\title{
Le fonds Antoine Meillet
}

Irène Fenoglio et Christian Puech

\section{(2) OpenEdition \\ Journals}

\section{Édition électronique}

URL : http://journals.openedition.org/genesis/1084

DOI : 10.4000/genesis. 1084

ISSN : 2268-1590

\section{Éditeur :}

Presses universitaires de Paris Sorbonne (PUPS), Société internationale de génétique artistique littéraire et scientifique (SIGALES)

\section{Édition imprimée}

Date de publication : 15 novembre 2012

Pagination : 189-194

ISBN : 978-2-84050-869-4

ISSN : 1167-5101

\section{Référence électronique}

Irène Fenoglio et Christian Puech, «L Le fonds Antoine Meillet », Genesis [En ligne], 35 | 2012, mis en ligne le 15 novembre 2014, consulté le 20 juin 2020. URL : http://journals.openedition.org/genesis/ 1084 ; DOI : https://doi.org/10.4000/genesis.1084 


\title{
Le fonds Antoine Meillet
}

\author{
Irène Fenoglio et Christian Puech
}

\section{Meillet dans son temps}

Antoine Meillet (1866-1936)1 ${ }^{1}$ prend la succession de Michel Bréal au Collège de France en 1906. Il va modeler de manière décisive la physionomie de la linguistique française jusqu'à sa mort et marquer plusieurs générations de son influence et de son autorité. Joseph Vendryes, Marcel Cohen et Émile Benveniste peuvent être considérés en France comme ses continuateurs les plus connus, même s'ils ont pu infléchir son enseignement dans des directions nouvelles. Ses cours à l'École pratique des hautes études et au Collège de France sont également assidûment suivis par de nombreux savants étrangers ${ }^{2}$.

À 40 ans (en 1906), cet « héritier » a étudié les langues classiques et orientales avec L. Havet, J. Darmesteter, A. Bergaigne, V. Henry, mais surtout S. Lévi, M. Bréal et $\mathrm{F}$. de Saussure à la Sorbonne, à l'École pratique des hautes études et au Collège de France.

Il devient à son tour un fondateur dont le rayonnement institutionnel et scientifique continue après son décès en 1936 et s'étend bien au-delà de la France. Héritier de ce que certains ont appelé « l'École linguistique de Paris », sa surface internationale va faire de lui, en effet, le fondateur d'une «École linguistique française » plus ou moins informelle et autopromue dont l'influence est sensible, par exemple, dans l'organisation du premier Congrès international des linguistes qui se tient à La Haye en 1928, à égale distance de Berlin et de Paris, dix années seulement après la fin de la Première Guerre mondiale (R. Jakobson, art. cit.).

C'est dire que la carrière d'Antoine Meillet parachève un premier tournant négocié antérieurement, essentiellement par M. Bréal et F. de Saussure, formés à la linguistique en Allemagne (mais aussi G. Paris, L. Meyer, V. Henry...), et qui ont fait de la Société linguistique de Paris l'un des centres de la linguistique européenne. Saussure, on le sait, enseigne à Paris à l'invitation de M. Bréal de 1881 à 1891. A. Meillet est d'abord l'un de ses élèves, puis son successeur en 1899-1900, jusqu'en 1917 à l'EPHE. Il joue donc un rôle capital, après le retour de Saussure à Genève en 1891, dans le renversement d'influence entre la linguistique allemande (hégémonique jusque-là) et le milieu parisien qu'il contribue à structurer par son autorité quasi sans partage.

Autrement dit, sa carrière assure la transition (longue et complexe) entre l'hégémonie de la grammaire historique et comparée et l'émergence d'une linguistique générale aux contours encore flous et disputés (S. Auroux (dir.), Histoire Épistémologie Langage, op. cit.).

De ce point de vue, le titre de l'ouvrage le plus connu de Meillet, Linguistique historique et linguistique générale, dit mieux que de longs discours une orientation à la fois scientifique, stratégique et institutionnelle. Si l'on

1. Sur Meillet on peut se référer à S. Auroux (dir.), Histoire Épistémologie Langage, «Antoine Meillet et la linguistique de son temps », vol. X, $\mathrm{n}^{\circ} 2$, 1988 [en particulier : S. Auroux, « La notion de linguistique générale », p. 37-56 et C. Puech et A. Radzinski, « Fait social et fait linguistique : A. Meillet et F. de Saussure », p. 75-84] ; G. Bergougnoux et Ch. de Lamberterie, Meillet aujourd'hui, Louvain/Paris, Peeters, 2006 ; J.-L. Chiss et C. Puech, Fondations de la linguistique, Bruxelles, Duculot, 1997 ; R. Jakobson, « The Twentieth Century in European and Amercican Linguistics », dans Selected Writings, Berlin/New York/Amsterdam, Mouton, 1985 ; A. Meillet, «L'état actuel des études de linguistique générale » [1906], dans A. Meillet, Linguistique générale et linguistique historique, t. I, Paris/Genève, Champion/Slatkine, 1921, [réed. 1982] ; C. Puech, «Antoine Meillet : quelle linguistique sociale ? », dans L'Opera scientifica di A. Meillet, a cura di A. Quattordio-Moreschini, Pisa, 1987 [réédité dans J.-L. Chiss et C. Puech, op. cit., 1997] ; P. Swiggers, «A. Meillet », dans Lexicon Grammaticorum, Tübingen, Max Niemeyer Verlag, 2009

2. Parmi lesquels Ch. Bally, H. Frei, G. Devoto, J. Kurylowicz, V. Brondal, A. Rosetti, A. Sommerfeldt... 
admet enfin que le Congrès international de 1928 voit s'affirmer un nouveau paradigme (structuraliste) avec le rôle central qu'y jouent les jeunes «pragois » Jakobson et Troubetzkoy, et avec la référence nouvelle accordée à F. de Saussure dans la « réformation » de la linguistique mondiale (Le Cours de linguistique générale est paru en 1916), on peut se représenter la figure de Meillet comme celle d'une « tête de réseau » de la linguistique mondiale.

Dans la carrière scientifique d'A. Meillet, on peut considérer que 1906 constitue une date charnière.

Jusque-là, le savant s'est essentiellement illustré dans le champ des études comparées indo-européennes avec une nette préférence pour la slavistique (sa thèse, en 1897 : Recherche sur l'emploi du génitiflaccusatif en vieux-slave) et l'arménien ancien et moderne, qu'il enseigne dans le cadre de l'École des langues orientales de 1902 à 1905. De cette période, on doit retenir sans doute l'ouvrage de référence qui va traverser le $\mathrm{xx}^{\mathrm{e}}$ siècle dans son domaine : l'Introduction à l'étude comparative des langues indo-européennes (1903) et son complément Les Dialectes indo-européens, qui paraîtra en $1908^{3}$.

L'expression des préoccupations de Meillet concernant la linguistique générale peut être datée de ce moment charnière.

À partir de 1906, les publications de Meillet vont suivre en effet un double cheminement parallèle : d'une part, la poursuite et l'amplification des travaux de grammaire historique et comparée des langues indoeuropéennes, mais aussi dans une moindre mesure sémitiques, caucasiennes, bantoues, amérindiennes, d'autre part, les réflexions générales sur des concepts, des méthodes, des principes valant pour la description et l'explication des faits linguistiques.

De ce point de vue, "L'avertissement ", tome I de Linguistique historique et linguistique générale (1921) résume bien l'intention du " généraliste »:

- l'ouvrage rassemble des articles et exposés publiés à partir de 1905 ;

- il vise un public divers qui n'est pas seulement celui des « spécialistes» du domaine mais inclut «l'homme cultivé », et surtout les savants des sciences connexes (philosophes, psychologues et sociologues);

- il cherche à modifier l'image que les pédagogues se font des langues et des faits linguistiques ;
- il s'inscrit enfin dans le long terme d'une évolution qui, du Moyen Âge à la fin du XviII e siècle, situait la « grammaire » dans le prolongement de la logique, tandis que le XIXe siècle, " en étendant aux faits psychiques et sociaux la méthode d'observation des faits qui est en usage dans les sciences physiques et naturelles depuis la Renaissance, a conduit à présenter la grammaire comme un ensemble de faits » (p. VIII, nous soulignons).

Ce quatrième point, dans son laconisme positiviste, mériterait d'être souligné. C'est que la linguistique générale de Meillet, celle qu'il promeut ${ }^{4}$, diffère sensiblement de celle que retiendra l'histoire de la linguistique la plus courante à partir du Cours de linguistique générale. Élève précoce de Saussure, A. Meillet contestera d'ailleurs la reconstruction (posthume) des cours (tardifs) du linguiste genevois par Ch. Bally et A. Sechehaye, dans lesquels, ditil, il ne retrouve pas les aperçus généraux dispensés par le Maître dès ses années parisiennes.

La généralité ne saurait être pour Meillet que le résultat d'une généralisation et la linguistique générale le résultat d'un dispositif largement pluridisciplinaire à l'édification duquel il contribue en linguiste, mais dont, pour cette raison, la réalisation devra être repoussée à plus tard... Tout au plus, ce " comparatisme historique généralisé » de Meillet devra-t-il préciser ce que le changement linguistique doit au système de la langue d'un côté, et au changement social de l'autre car, « [...] du fait que le langage est une institution sociale, il résulte que la linguistique est une science sociale, et le seul élément variable auquel on puisse recourir pour rendre compte du changement linguistique est le changement social dont les variations du langage ne sont que les conséquences parfois immédiates et directes, et le plus souvent médiates et indirectes » (1906, art. cit., p. 17).

3. Mais on ne doit pas oublier les nombreuses publications dans les revues européennes les plus prestigieuses : le Bulletin de la Société linguistique de Paris, les Indogermanische Forschungen et le Journal asiatique, sur des sujets qui peuvent concerner l'indo-iranien, le grec, le latin et les langues celtiques...

4. Et celle qui lui sert à évaluer d'autres projets dans le Bulletin de la Société linguistique de Paris, dans des revues de psychologie, dans Sciencia mais surtout dans L'Année sociologique de Durkheim et Mauss. 
C'est là l'objet de sa leçon inaugurale de grammaire comparée au Collège de France en 1906. Nous présentons, en illustration, un des premiers feuillets du brouillon ${ }^{5}$ de cette leçon inaugurale (fig. 4).

\section{Les manuscrits d'Antoine Meillet}

Les manuscrits d'Antoine Meillet sont conservés à l'IMEC, mais proviennent des archives du Collège de France6.

Dans vingt-quatre boîtes d'archives, le fonds comprend des manuscrits de travail (préparations d'articles ou de cours), de très nombreux plans d'articles, de livres, mais aussi des publications en tirés à part, une importante correspondance, dont la part la plus intéressante comprend des lettres reçues des plus grands linguistes de son temps : Ferdinand de Saussure, Émile Benveniste, Marcel Cohen, Joseph Vendryes, Lucien Tesnière. On y trouve également de nombreux carnets de notes, un récit de voyage en Arménie, des coupures de presse et des archives familiales, ainsi qu'un ensemble de documents postérieur au décès de l'auteur.

Aucun véritable travail n'a encore été effectué sur ce fonds d'archives, impressionnant à plusieurs titres.

Outre son ampleur et son intérêt pour l'histoire de la linguistique, il confronte le généticien à plusieurs questions.

Se posent, tout d'abord, de sérieux problèmes de lecture : Antoine Meillet est illisible. Son écriture minuscule et rapide épuise notre attention et, par ailleurs - et malheureusement -, l'ancienneté de ses papiers fait qu'ils sont pour certains quasiment « intouchables » au risque de tomber en poussière. Aujourd'hui, seule la numérisation pourra les sauver.

Mais l'observation de ces manuscrits ouvre une autre question fort intéressante, celle des habitus de travail et plus précisément celle de la transmission de ces habitus. Nous avons ouvert les manuscrits d'Antoine Meillet après avoir ouvert ceux d'Émile Benveniste qui se trouvent au département des Manuscrits de la BnF, et nous remarquons une façon semblable chez les deux linguistes de procéder, par exemple, à l'écriture d'un article : un ensemble de notes sur tout support, de tout format, une première rédaction (premier brouillon),
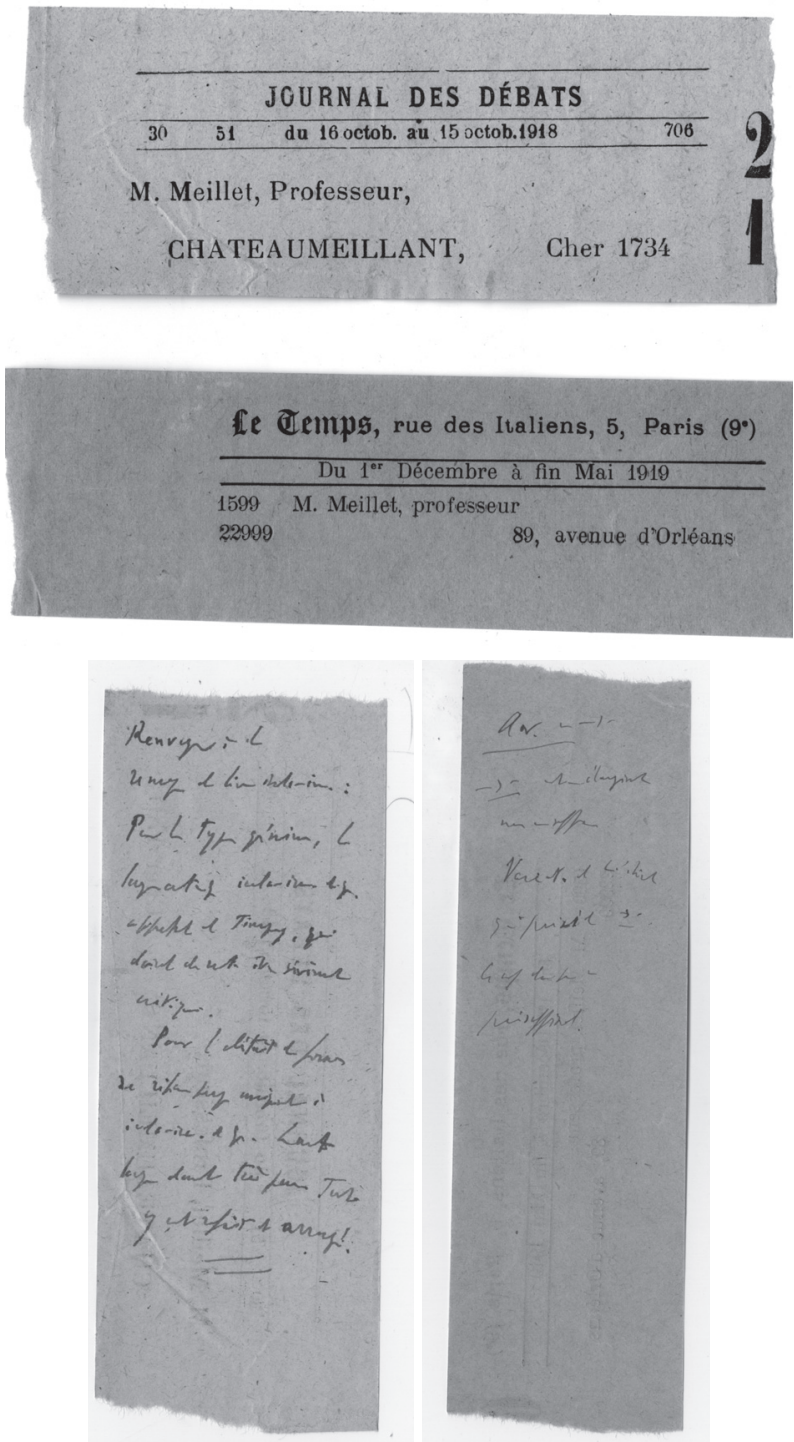

Fig. 1 : Notes inscrites sur le verso de bandeaux de journaux (Fonds Antoine Meillet/Collège de France/IMEC)

souvent un second brouillon mis au net 7 . Certes, il sera nécessaire de multiplier les études comparatives entre plusieurs processus de différents linguistes pour pouvoir

5. L'édition de cette leçon inaugurale se trouve dans l'ouvrage Linguistique historique et linguistique générale de Meillet, Paris, Champion, 1921.

6. À la suite du contrat de dépôt établi en 2001 avec l'IMEC, ces archives ont été transférées à l'Abbaye d'Ardenne après un premier récolement effectué entre novembre 2001 et janvier 2002. Simon Bouquet a réalisé un premier inventaire de ce fonds, alors qu'il se trouvait encore au Collège de France. C'est cet inventaire qui a été repris par l'IMEC.

7. Voir, dans le présent volume, l'article « Genèse du geste linguistique : une complexité heuristique ». 

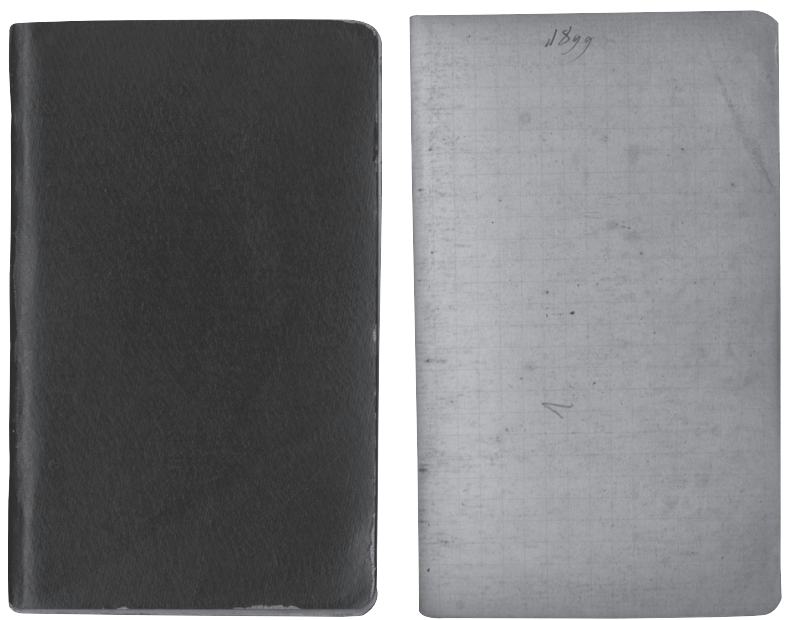

Fig. 2 : Carnet de poche de l'année 1899

(Fonds Antoine Meillet/Collège de France/IMEC)

tirer des conclusions, mais cette reconnaissance chez Meillet d'une même façon de faire que Benveniste, quand l'on sait que Benveniste a été l'élève de Meillet, doit nous alerter sur la question de la transmission des façons d'écrire, sur la transmission des acquis ou des automatismes de savoir-faire socialement constitués, sur l'histoire des «apprentissages » de l'écriture scientifique, et sur ce point, la richesse des fonds d'archives est incontournable.

Restons-en à Meillet. Dans un processus général que nous venons de décrire, Meillet a des habitudes qui lui sont particulières. Les notes préparatoires sont inscrites sur toutes sortes de bouts de papier et notamment, de façon récurrente, sur des bandeaux d'envois de journaux : Le Temps, Le Journal des débats, et même L'Action française (fig. 1).

Nous en trouvons énormément dans ses archives. Par exemple, dans un dossier de notes et brouillons pour la «Conférence sur les principes de la linguistique historique » (décembre 1910), les notes préparatoires à la rédaction de la conférence se retrouvent entre les feuillets rédigés, sortes de paperoles libres mais demeurées à leur place d'insertion. Il s'agit là aussi d'une disposition particulière à Meillet, comparativement à Benveniste pour lequel on retrouve les notes dans un ensemble séparé de la rédaction.
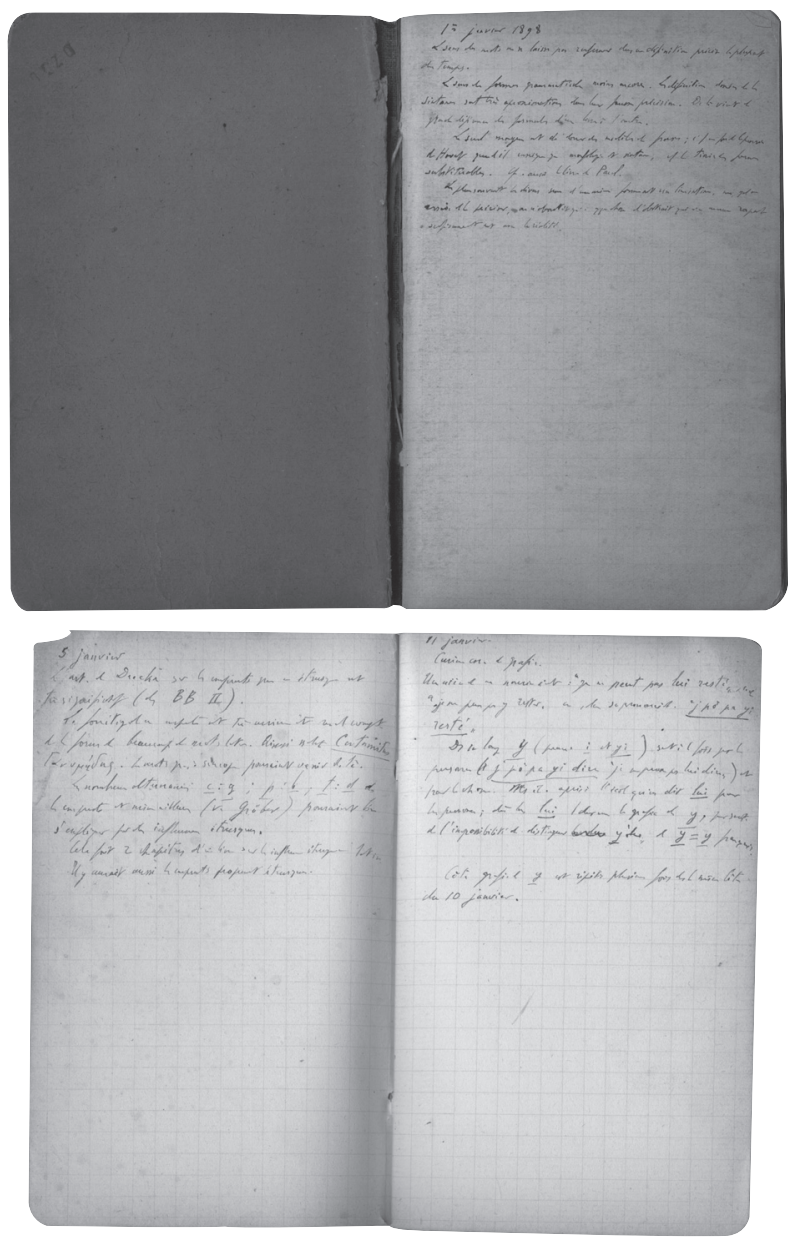

Fig. 3 : Exemples de pages de carnet de poche tenant lieu de journal de travail

(Fonds Antoine Meillet/Collège de France/IMEC)

Le travail rédactionnel s'effectue, pour Meillet, la plupart du temps sur des feuilles de papier blanc (aujourd'hui jauni) assez épais de grande qualité. La plupart du temps, les brouillons s'écrivent au verso d'un papier déjà utilisé pour le brouillon d'un autre essai ou cours, il barre alors verticalement la partie écrite (qui devient le recto). Ces feuilles sont pliées en deux, en leur milieu, dans le sens de la hauteur : la rédaction se poursuit à droite, la colonne de gauche étant réservée aux reprises et ajouts. Signalons encore que nous trouvons de nombreux plans d'articles dans ses papiers. 


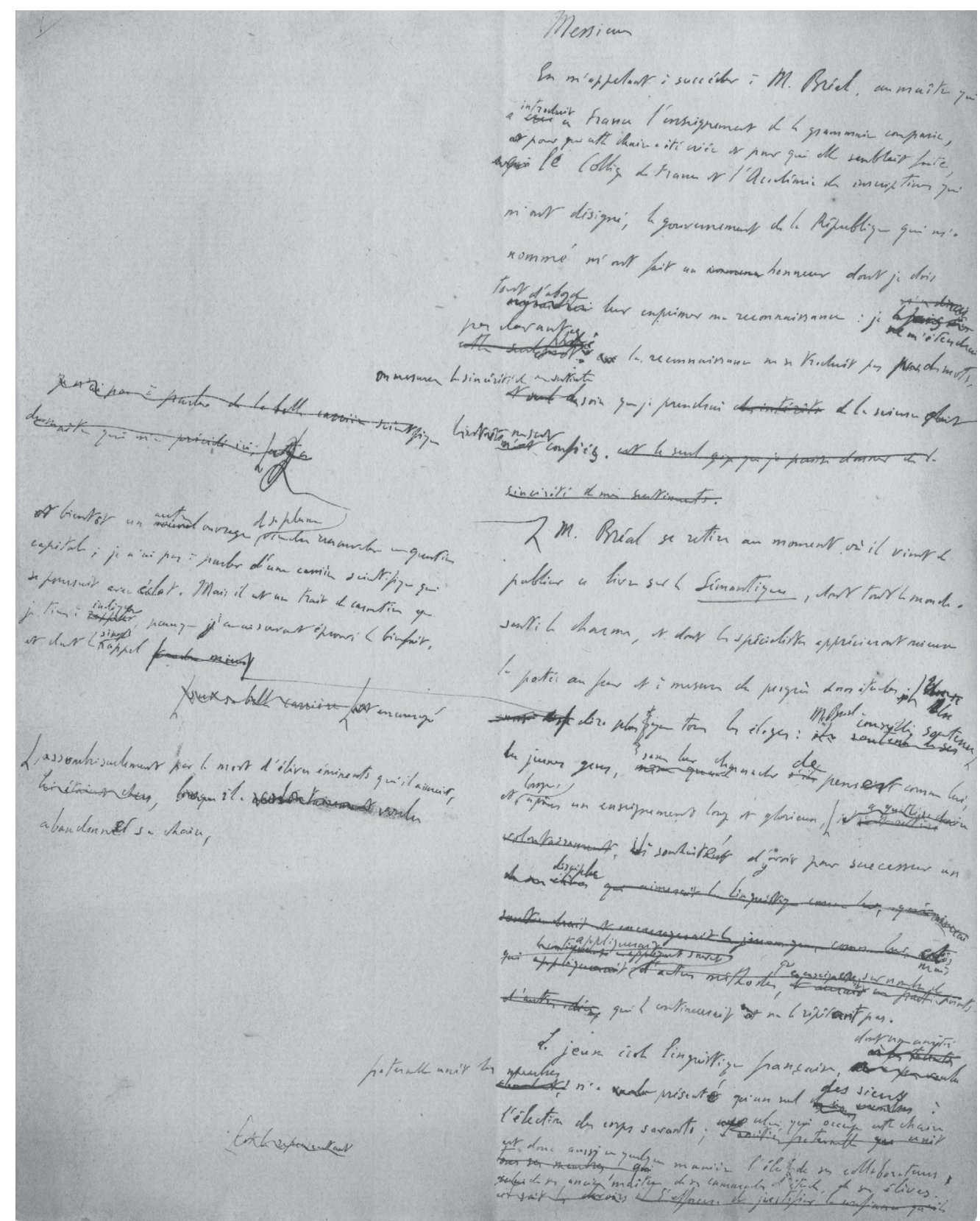

Fig. 4 : Feuillet 3 du brouillon de la leçon d'ouverture du cours de grammaire comparée d'A. Meillet au Collège de France (13 mars 1906), intitulée « L'état actuel des études de linguistique générale »

(Fonds Antoine Meillet/Collège de France/IMEC) 
À côté des dossiers complexes relatifs à l'écriture de textes, Meillet remplissait des séries de petits carnets de poche $(20 \mathrm{~cm} \times 14 \mathrm{~cm})$, à la couverture en moleskine noire. Il s'agit de journaux de travail, un carnet par année dont le chiffre est inscrit sur la première page. Lorsque le carnet n'est pas terminé, il est laissé avec ses pages blanches et un autre carnet est ouvert pour l'année suivante (fig. 2).
Ces carnets portent, sous la mention de la date, toutes sortes d'observations : des notations linguistiques comme des remarques générales ou des réflexions beaucoup plus personnelles comme : « travail médiocre », « travail mou, très mou », « travail satisfaisant » (fig. 3).

Nous avons pu recenser près de quarante-cinq carnets de ce type dans le fonds. Leurs potentialités d'exploitation en relation avec les dossiers avant-textuels des écrits du linguiste sont immenses.

Irène Fenoglio dirige, au sein de l'ITEM, l'équipe « Génétique du texte et théories linguistiques ». Ses travaux portent essentiellement sur les manuscrits de linguistes. Dernières publications : «Déplier l'écriture pensante pour relire l'article publié. Les manuscrits de "L'appareil formel de l'énonciation" ", dans Relire Benveniste. Réceptions actuelles des Problèmes de linguistique générale (dir. E. Brunet et R. Mahrer, Academia) ; Émile Benveniste, Dernières leçons. Collège de France 1968 et 1969 (texte établi avec J.-C. Coquet, EHESS/Gallimard/Seuil, 2012).

irene.fenoglio@ens.fr

Christian Puech est professeur des Universités à l'université Sorbonne Nouvelle-Paris III. Spécialiste d'histoire des théories linguistiques, il est l'auteur de nombreux articles. Dernière publication dirigée : « Histoire des idées linguistiques et horizons de rétrospection », Histoire Épistémologie Langage, n² 28/1, 2006.

christian.puech@univ-paris3.fr 